\title{
УДК 621.77
}

Михалевич В. М., д.т.н.; Краєвський В. О., к.т.н.; Добранюк Ю. В., к.т.н. Вінницький національний технічний університет, Вінниця, Україна

\section{УДОСКОНАЛЕННЯ МЕТОДУ РОЗВ'ЯЗУВАННЯ ДВОВИМІРНОЇ ЗАДАЧІ ПРЕСУВАННЯ ШТАБИ}

Mykhalevych V. M., Kraevskiy V. O., Dobraniuk Yu. V.

Vinnytsya National Technical University, Vinnytsya, Ukraine

\section{ANALYSIS OF THE SYSTEM OF DIFFERENTIAL EQUATIONS IN SOLVING TWO- DIMENSIONAL PROBLEM PRESSING OF THE STRIP}

Проаналізовано метод розв'язання двовимірної задачі пресування штаби з метою удосконалення та розробки комп'ютерної підтримки його використання. В результаті досліджень, в рамках використання моделі нелінійно-в'язкого тіла, було встановлено структуру залежностей інтенсивності напружень, накопиченої деформації та безрозмірного показника напруженого стану від радіуса та полярного кута. Запропоновано нові прийоми отримання відомих залежностей у вигляді системи двох звичайних нелінійних диференціальних рівнянь та зведення цієї системи до нелінійного диференціального рівняння 2-го порядку із змінними коефіцієнтами. Розроблено відповідні інформаційні Марlе-технології.

Ключові слова: пресування штаби, двовимірна задача, напружено-деформований стан, накопичена деформачія, рівняння стану.

Сучасні потужні обчислювальні комплекси, зокрема DEFORM 3D, QFORM, ANSYS та інші. надають можливість отримати розв'язки широкого кола задач визначення напружено-деформованого стану матеріалів під час пластичного деформування. Проте всі ці пакети є комерційними, що суттєво обмежує їх практичне використання. Тому актуальним $\epsilon$ аналіз та удосконалення відомих методів визначення напруженодеформованого стану матеріалів, а також розробка інформаційних технологій для підвищення ефективності використання цих методів. Крім цього такі методи використовуються як тестові задачі для апробації достовірності результатів, що отримуються за допомогою зазначених обчислювальних комплексів. Ускладнення та урізноманітнення тестових задач сприятиме і вдосконаленню цих комплексів.

Метою цієї статті є удосконалення методу розв'язування двовимірної задачі пресування штаби на основі аналізу системи диференціальних рівнянь та розробки комп’ютерної підтримки його використання.

Розглядатимемо розв'язання двовимірної задачі пресування штаби, що базується на дослідженні течії матеріалу у клиновидному каналі. Надалі акценти ставитимемо на висвітленні окремих важливих деталей, яким в [1] приділено недостатньо уваги.

Постановка задачі базується на таких основних припущеннях:

1. Течія матеріалу є радіальною.

2. Властивості матеріалу описуються моделлю нелінійно-в'язкого тіла.

У загальному випадку плоскої деформації залежності швидкостей деформацій $\dot{\xi}_{\rho}, \dot{\xi}_{t}, \gamma_{\rho t}$ від швидкостей переміщення $v_{\rho}, v_{t}$ в циліндричних координатах мають такий вигляд $[1,2]$

$$
\begin{gathered}
\dot{\xi}_{\rho}=\frac{\partial v_{\rho}}{\partial \rho}, \quad \dot{\xi}_{t}=\frac{1}{\rho} \frac{\partial v_{t}}{\partial \alpha}+\frac{v_{\rho}}{\rho}, \\
\gamma_{\rho t}=\rho \frac{\partial}{\partial \rho}\left(\frac{v_{t}}{\rho}\right)+\frac{1}{\rho} \frac{\partial v_{\rho}}{\partial \alpha}
\end{gathered}
$$

Припущення про радіальну течію: $v_{t}=0, v_{\rho}=v(\rho, \alpha)$, надає можливість спростити останні співвідношення

$$
\dot{\xi}_{\rho}=\frac{\partial v(\rho, \alpha)}{\partial \rho}, \quad \dot{\xi}_{t}=\frac{v(\rho, \alpha)}{\rho}, \quad \dot{\gamma}=\dot{\gamma}_{\rho t}=\frac{1}{\rho} \frac{\partial v(\rho, \alpha)}{\partial \alpha} .
$$

Оскільки розглядається плоска деформація $\left(\dot{\xi}_{z}=0\right)$ умова нестисливості у швидкостях деформації набуває вигляду 


$$
\dot{\xi}_{\rho}=-\dot{\xi}_{t} .
$$

Звідки, з урахуванням (2), випливає диференціальне рівняння для швидкостей радіального переміщення

$$
\frac{\partial v(\rho, \alpha)}{\partial \rho}+\frac{v(\rho, \alpha)}{\rho}=0
$$

Маємо диференціальне рівняння з відокремлюваними змінними. Відокремимо змінні та проінтегруємо

$$
\int \frac{\partial v}{v}=-\int \frac{\partial \rho}{\rho}+\ln (-\omega(\alpha))
$$

або

$$
v(\rho, \alpha)=-\frac{\omega(\alpha)}{\rho}
$$

де $\omega=\omega(\alpha)$ - невідома функція.

Знак мінус у останньому співвідношенні відображує той факт, що течія матеріалу відбувається у напрямі, протилежному $\rho$. 3 урахуванням (6) співвідношення (2) набувають вигляду

$$
\dot{\xi}_{\rho}=\frac{\omega(\alpha)}{\rho^{2}}, \quad \dot{\xi}_{t}=-\frac{\omega(\alpha)}{\rho^{2}}, \quad \dot{\gamma}=-\frac{\omega^{\prime}(\alpha)}{\rho^{2}} .
$$

Отже, припущення про радіальну течію надає можливість установити залежність компонентів швидкостей деформацій від радіуса.

Вираз для інтенсивності швидкостей деформацій

$$
\dot{\xi}_{i}=\sqrt{2 \cdot \dot{\xi}_{i j} \cdot \dot{\xi}_{i j}}=\sqrt{\frac{2}{3}} \cdot \sqrt{\dot{\xi}_{\rho}^{2}+\dot{\xi}_{t}^{2}+\dot{\xi}_{z}^{2}+\frac{1}{2} \cdot\left(\dot{\gamma}_{\rho t}^{2}+\dot{\gamma}_{t z}^{2}+\dot{\gamma}_{z \rho}^{2}\right)}
$$

із урахуванням плоскої деформації та умови нестисливості (3) матиме вигляд

$$
\dot{\xi}_{i}=\frac{\sqrt{3}}{3} \cdot \sqrt{4 \cdot \dot{\xi}_{\rho}^{2}+\dot{\gamma}^{2}} .
$$

а з урахуванням (7) отримаємо

$$
\dot{\xi}_{i}=\frac{\sqrt{3}}{3 \cdot \rho^{2}} \cdot \sqrt{4 \cdot \omega^{2}(\alpha)+\left(\omega^{\prime}(\alpha)\right)^{2}}
$$

Уведемо нову функцію $g(\alpha)$

$$
g(\alpha)=\sqrt{4 \cdot \omega^{2}(\alpha)+\left(\omega^{\prime}(\alpha)\right)^{2}}
$$

тоді

$$
\dot{\xi}_{i}(\alpha, \rho)=\frac{\sqrt{3}}{3} \cdot \frac{g(\alpha)}{\rho^{2}} .
$$

За умов плоскої деформації для компонентів тензора напружень виконуються такі співвідношення

$$
\sigma_{z}=\frac{\sigma_{\rho}+\sigma_{t}}{2}, \quad \tau_{z \rho}=0, \quad \tau_{z t}=0 .
$$

З урахуванням цих співвідношень інтенсивність напружень

$$
\sigma_{i}=\sqrt{\frac{3 \cdot s_{i j} \cdot s_{i j}}{2}}=\frac{\sqrt{2}}{2} \cdot \sqrt{\left(\sigma_{\rho}-\sigma_{t}\right)^{2}+\left(\sigma_{z}-\sigma_{t}\right)^{2}+\left(\sigma_{z}-\sigma_{\rho}\right)^{2}+6 \cdot\left(\tau_{\rho t}^{2}+\tau_{z t}^{2}+\tau_{z \rho}^{2}\right)}
$$

набуває вигляду

$$
\sigma_{i}=\frac{\sqrt{3}}{2} \cdot \sqrt{\left(\sigma_{\rho}-\sigma_{t}\right)^{2}+4 \cdot \tau^{2}}
$$

де $s_{i j}-$ компоненти девіатора напружень; $\tau=\tau_{\rho t}$.

Відповідно до другого припущення рівняння стану представимо у вигляді [1]

$$
\sigma_{i}=a \cdot \dot{\xi}_{i}^{m_{1}} \cdot \varepsilon_{i}^{m_{2}} .
$$

де $a, m_{1}, m_{2}$ - матеріальні константи; накопичена деформація або параметр Удквіста $\varepsilon_{i}$ визначається інтегруванням вздовж шляху деформування

$$
\varepsilon_{i}=\int_{0}^{T} \dot{\xi}_{i}(\alpha(T), \rho(T)) \cdot d T .
$$

Тут $T$ - час протягом якого накопичується деформація макрочастинки. 
3 урахуванням (12) та гіпотези про радіальну течію, співвідношення для накопиченої деформації матиме такий вигляд

$$
\varepsilon_{i}=\frac{\sqrt{3}}{3} \cdot g\left(\alpha_{j}\right) \cdot \int_{0}^{T} \frac{d T}{\rho^{2}(T)} .
$$

Враховуючи, що

$$
d \rho=v\left(\rho, \alpha_{j}\right) \cdot d T
$$

з урахуванням (6), матимемо

$$
\varepsilon_{i}\left(\rho, \alpha_{j}\right)=\frac{\sqrt{3}}{3} \cdot \frac{g\left(\alpha_{j}\right)}{\omega\left(\alpha_{j}\right)} \cdot \ln \left(\frac{\rho_{s}\left(\alpha_{j}\right)}{\rho\left(\alpha_{j}\right)}\right),
$$

де $\rho_{s}$ - радіус початку зони пластичної деформації $\left(\rho_{s}>\rho\right)$.

Рівняння стану (16) з урахуванням (12) та (20) набуває вигляду

$$
\sigma_{i}=\left(\frac{\sqrt{3}}{3} \cdot g(\alpha)\right)^{m_{1}+m_{2}} \cdot \frac{a}{\rho^{2 \cdot m_{1}}} \cdot\left(\frac{\ln \left(\frac{\rho_{s}(\alpha)}{\rho(\alpha)}\right)}{\omega(\alpha)}\right)^{m_{2}} .
$$

В окремому випадку $m_{2}=0, m_{1}=m$ співвідношення (21) стає тотожним залежності, що наведена у [1]

$$
\sigma_{i}=a \cdot\left(\frac{\sqrt{3} \cdot g(\alpha)}{3 \cdot \rho^{2}}\right)^{m} .
$$

Відомо, що компоненти девіатора напружень у випадку плоскої деформації можна представити такими залежностями [2]

$$
\left.\begin{array}{l}
s_{\rho}=\frac{\sqrt{3}}{3} \cdot \sigma_{i} \cdot \cos (2 \cdot \varphi(\rho, \alpha)) \\
s_{t}=-\frac{\sqrt{3}}{3} \cdot \sigma_{i} \cdot \cos (2 \cdot \varphi(\rho, \alpha)) \\
\tau=\frac{\sqrt{3}}{3} \cdot \sigma_{i} \cdot \sin (2 \cdot \varphi(\rho, \alpha))
\end{array}\right\},
$$

де $\varphi=\varphi(\rho, \alpha)$ - деяка невідома функція. За цих умов співвідношення (15) перетворюється на тотожність.

Використовуватимемо залежності компонентів швидкостей деформацій повзучості від компонентів напружень у вигляді [1]

$$
\dot{\xi}_{i j}=\frac{3}{2} \cdot \frac{\dot{\xi}_{i}}{\sigma_{i}} \cdot\left(\sigma_{i j}-\delta_{i j} \cdot \sigma_{0}\right),
$$

де $\sigma_{0}=\sigma_{0}(\rho, \alpha)$ - середнє нормальне напруження; $\delta_{i j}$ - символ Кронекера.

Останні співвідношення передбачають припущення про ізотропність матеріалу та незалежність повзучості від першого інваріанта тензора напружень та третього інваріанта девіатора напружень. В якості еквівалентної швидкості деформацій та еквівалентного напруження прийнято інтенсивність швидкостей деформацій та інтенсивність напружень відповідно.

На основі (24) з урахуванням (23) отримаємо

$$
\left.\begin{array}{l}
\dot{\xi}_{\rho}=\frac{\sqrt{3}}{2} \cdot \dot{\xi}_{i}(\rho, \alpha) \cdot \cos (2 \cdot \varphi(\rho, \alpha)) \\
\dot{\xi}_{t}=-\frac{\sqrt{3}}{2} \cdot \dot{\xi}_{i}(\rho, \alpha) \cdot \cos (2 \cdot \varphi(\rho, \alpha)) \\
\dot{\gamma}=\sqrt{3} \cdot \dot{\xi}_{i}(\rho, \alpha) \cdot \sin (2 \cdot \varphi(\rho, \alpha))
\end{array}\right\} .
$$

Зіставленням виразів для $\dot{\xi}_{\rho}$ та $\dot{\gamma}$ на основі (7) та (25) з урахуванням (12), а також

$$
\dot{\gamma}=\dot{\gamma}_{\rho t}=2 \cdot \dot{\xi}_{\rho t} .
$$

отримаємо 


$$
\begin{aligned}
\omega(\alpha) & =\frac{g(\alpha)}{2} \cdot \cos (2 \cdot \varphi(\rho, \alpha)) . \\
\omega^{\prime}(\alpha) & =-g(\alpha) \cdot \sin (2 \cdot \varphi(\rho, \alpha)) .
\end{aligned}
$$

Із співвідношень (26), (27) випливає, що функція $\varphi$ залежить тільки від змінної $\alpha$

$$
\varphi=\varphi(\alpha) \text {. }
$$

Диференціальні рівняння рівноваги елемента, що вирізаний з штаби двома радіальними перетинами під кутом $\alpha$ та двома коловими перетинами радіусів $\rho$ та $\rho+d \rho$, запишемо у зміненому в порівнянні 3 [2] вигляді

$$
\left.\begin{array}{l}
\frac{\partial s_{\rho}}{\partial \rho}+\frac{\partial \sigma_{0}}{\partial \rho}+\frac{1}{\rho} \cdot \frac{\partial \tau}{\partial \alpha}+\frac{s_{\rho}-s_{t}}{\rho}=0 \\
\frac{\partial \tau}{\partial \rho}+\frac{1}{\rho} \cdot \frac{\partial s_{t}}{\partial \alpha}+\frac{1}{\rho} \cdot \frac{\partial \sigma_{0}}{\partial \alpha}+\frac{2 \cdot \tau}{\rho}=0
\end{array}\right\}
$$

Перепишемо (23) з урахуванням (22), (29)

$$
\left.\begin{array}{l}
s_{\rho}=C_{1} \cdot \frac{G_{1}(\alpha)}{\rho^{2 \cdot m}}, \\
s_{t}=-C_{1} \cdot \frac{G_{1}(\alpha)}{\rho^{2 \cdot m}}, \\
\tau=C_{1} \cdot \frac{G_{2}(\alpha)}{\rho^{2 \cdot m}},
\end{array}\right\},
$$

де $-G_{1}=g^{m}(\alpha) \cdot \cos (2 \cdot \varphi(\alpha)), \quad G_{2}=g^{m}(\alpha) \cdot \sin (2 \cdot \varphi(\alpha)), \quad C_{1}=a \cdot\left(\frac{\sqrt{3}}{3}\right)^{m+1}$.

Розпишемо перше рівняння із системи диференціальних рівнянь (30) з урахуванням (31). Для цього скористаємося системою комп'ютерної математики (СКМ) Maple:

restart:

\# Введемо позначення

G1:=g(alpha $)^{\wedge} \mathbf{m}^{*} \cos (2 *$ phi(alpha $\left.)\right)$;

G2:=g(alpha $)^{\wedge} \mathrm{m} * \sin (2 *$ phi(alpha $\left.)\right)$;

$C 1:=a^{*}\left(1 / 3^{*} 3^{\wedge}(1 / 2)\right)^{\wedge}(m+1)$;

$$
\begin{gathered}
G_{1}:=g(\alpha)^{m} \cos (2 \varphi(\alpha)) . \\
G_{2}:=g(\alpha)^{m} \sin (2 \varphi(\alpha)) . \\
C_{1}:=a\left(\frac{\sqrt{3}}{3}\right)^{(m+1)}
\end{gathered}
$$

\# Вирази для компонет девіатора напружень відповідно до (31) та введених позначень S[rho]:=C[1]*G[1](alpha) $/$ rho^$^{\wedge}(2 * m)$;

$\mathrm{S}[\mathrm{t}]:=-\mathrm{C}[1] * \mathrm{G}[1]($ alpha $) / \mathrm{rho}^{\wedge}(2 * \mathrm{~m})$; tau: $=\mathrm{C}[1] * \mathrm{G}[2]($ alpha $) / \mathrm{rho}^{\wedge}(2 * \mathrm{~m})$;

$$
\begin{aligned}
s_{\rho} & :=\frac{G_{1} C_{1}(\alpha)}{\rho^{(2 m)}} \\
s_{t} & :=-\frac{C_{1} G_{1}(\alpha)}{\rho^{(2 m)}} \\
\tau & :=\frac{C_{1} G_{2}(\alpha)}{\rho^{(2 \cdot m)}}
\end{aligned}
$$

\# Перше із системи диференціальних рівнянь (30)

$\operatorname{diff}(\mathrm{S}[\mathbf{r h o}], \mathbf{r h o})+\operatorname{diff}(\operatorname{sigma}[0]($ rho,alpha $)$, rho $)+\operatorname{diff}($ tau,alpha)/rho+(S[rho]-S $[\mathbf{t}]) / \mathbf{r h o}=0$;

\# Помножимо обидві частини рівняння на знаменник першого дробу

$\% *\left(\right.$ rho $\left.^{\wedge}(2 * \mathbf{m}) * \mathbf{r h o}\right)$;

\# Розкриємо дужки

$\operatorname{expand}(\%, \mathbf{m}+1)$

\# Зведемо подібні

$\operatorname{collect}(\%,[a, C[1], G[1]($ alpha $)])$; 
\# Здійснимо перетворення combine(\%,power);

$$
\begin{gathered}
-\frac{2 G_{1} C_{1}(\alpha) m}{\rho^{(2 m)} \rho}+\left(\frac{\partial}{\partial \rho} \sigma_{0}(\rho, \alpha)\right)+\frac{G_{1}\left(\frac{d}{d \alpha} G_{2(\alpha)}\right)}{\rho^{(2 m)} \rho}+\frac{2 G_{1} C_{1}(\alpha)}{\rho^{(2 m)} \rho}=0 \\
\left(-\frac{2 G_{1} C_{1}(\alpha) m}{\rho^{(2 m)} \rho}+\left(\frac{\partial}{\partial \rho} \sigma_{0}(\rho, \alpha)\right)+\frac{G_{1}\left(\frac{d}{d \alpha} G_{2(\alpha)}\right)}{\rho^{(2 m)} \rho}+\frac{2 G_{1} C_{1}(\alpha)}{\rho^{(2 m)} \rho}\right) \rho^{(2 m)} \rho=0 \\
-2 G_{1} C_{1}(\alpha) m+\left(\rho^{m}\right)^{2} \rho\left(\frac{\partial}{\partial \rho} \sigma_{0}(\rho, \alpha)\right)+G_{1}\left(\frac{d}{d \alpha} G_{2(\alpha)}\right)+2 G_{1} C_{1}(\alpha)=0 \\
\left((-2 m+2) C_{1}(\alpha)+\left(\frac{d}{d \alpha} G_{2}(\alpha)\right)\right) C_{1}+\left(\rho^{m}\right)^{2} \rho\left(\frac{\partial}{\partial \rho} \sigma_{0}(\rho, \alpha)\right)=0 \\
\left((-2 m+2) C_{1}(\alpha)+\left(\frac{d}{d \alpha} G_{2}(\alpha)\right)\right) C_{1}+\left(\frac{\partial}{\partial \rho} \sigma_{0}(\rho, \alpha)\right) \rho^{(2 m+1)}=0
\end{gathered}
$$

Останній вираз тотожний отриманому в [1], що запишемо у вигляді:

$$
\frac{\partial \sigma_{0}(\rho, \alpha)}{\partial \rho}=\frac{C_{1}}{\rho^{2 \cdot m+1}} \cdot\left(2 \cdot(m-1) \cdot G_{1}(\alpha)-\frac{d G_{2}(\alpha)}{\partial \alpha}\right)
$$

За аналогією з першим, розпишемо друге із системи диференціальних рівнянь (30):

\# Друге із системи диференціальних рівнянь (30)

$\operatorname{diff}($ tau,rho $)+\operatorname{diff}(\mathrm{S}[\mathrm{t}]$, alpha) $/ \mathrm{rho}+\operatorname{diff}(\operatorname{sigma}[0]($ rho,alpha $)$, alpha) $/ \mathbf{r h o}+(2 * \operatorname{tau}) / \mathbf{r h o}=\mathbf{0}$;

$\% *\left(\mathbf{r h o}^{\wedge}(2 * \mathrm{~m}) * \mathbf{r h o}\right)$ :

$\operatorname{expand}(\%, \mathbf{m}+1)$ :

$\operatorname{collect}(\%,[\mathbf{a}, \mathrm{C}[1], \mathrm{G}[2]($ alpha) $])$ :

combine(\%,power);

$$
\begin{gathered}
-\frac{2 G_{1} C_{2}(\alpha) m}{\rho^{(2 m)} \rho}-\frac{G_{1}\left(\frac{d}{d \alpha} G_{1(\alpha)}\right)}{\rho^{(2 m)} \rho}+\frac{\frac{\partial}{\partial \alpha} \sigma_{0}(\rho, \alpha)}{\rho}+\frac{2 G_{1} C_{2}(\alpha)}{\rho^{(2 m)} \rho}=0 \\
\left((-2 m+2) C_{2}(\alpha)-\left(\frac{d}{d \alpha} G_{1}(\alpha)\right)\right) C_{1}+\rho^{(2 m)}\left(\frac{\partial}{\partial \alpha} \sigma_{0}(\rho, \alpha)\right)=0
\end{gathered}
$$

Останній вираз з урахуванням уведених позначень тотожний отриманому в [1], що запишемо у вигляді:

$$
\frac{\partial \sigma_{0}(\rho, \alpha)}{\partial \alpha}=\frac{C_{1}}{\rho^{2 \cdot m}} \cdot\left(\frac{d G_{1}(\alpha)}{\partial \alpha}+2 \cdot(m-1) \cdot G_{2}(\alpha)\right) .
$$

Відокремимо змінні в диференціальному рівнянні (34) та проінтегруємо

$$
\int d \sigma_{0}(\rho, \alpha)=\int \frac{C_{1}}{\rho^{2 \cdot m+1}} \cdot\left(2 \cdot(m-1) \cdot G_{1}(\alpha)-\frac{d G_{2}(\alpha)}{\partial \alpha}\right) \cdot d \rho+b,
$$

або

$$
\sigma_{0}(\rho, \alpha)=\frac{C_{1}}{2 \cdot m \cdot \rho^{2 \cdot m}} \cdot\left(2 \cdot(m-1) \cdot G_{1}(\alpha)-\frac{d G_{2}(\alpha)}{\partial \alpha}\right)+b
$$

де $b=$ const .

Для більш повного аналізу напруженого стану, крім напружень необхідно визначити показники напруженого стану. Широке застосування отримав показник $[3,4,5,6,7]$

$$
\eta=\frac{3 \cdot \sigma_{0}}{\sigma_{i}}
$$

На основі (37), (22) отримаємо

$$
\eta=\frac{\sqrt{3}}{2 \cdot m \cdot g(\alpha)^{m}} \cdot\left(2 \cdot(m-1) \cdot G_{1}(\alpha)-\frac{d G_{2}(\alpha)}{\partial \alpha}\right)+\frac{b}{a} \cdot\left(\frac{\sqrt{3} \cdot g(\alpha)}{3 \cdot \rho^{2}}\right)^{-m} .
$$

При $b=0$ цей показник не залежить від радіуса $\rho$, що потребує окремого дослідження. 
Продиференціюємо рівняння (34) за змінною $\alpha$, рівняння (35) за змінною $\rho$. Оскільки ліві частини отриманих рівностей тотожні, прирівняємо праві частини та виконаємо елементарні перетворення. Здійснимо вказані операції за допомогою СКМ Maple:

\# Диференціювання за змінною $\alpha$

$\operatorname{diff}(\operatorname{diff}($ sigma $[0]($ rho,alpha),rho) $=-((-$

$2 * m+2) * G[1]($ alpha $)+\operatorname{diff}(G[2]($ alpha $)$, alpha $)) * C[1] /\left(\mathbf{r h o}^{\wedge}(2 * m+1)\right)$, alpha $) ;$

\# Диференціювання за змінною $\rho$

$\operatorname{diff}\left(\operatorname{diff}\left(\operatorname{sigma}[0]\left(\right.\right.\right.$ rho,alpha),alpha) $=-((-2 * m+2) * G[2]($ alpha $)-\operatorname{diff}(G[1]($ alpha $)$, alpha $)) * C[1] /\left(\operatorname{rho} 0^{\wedge}(2 * m)\right)$, rho $)$;

\# Прирівнювання правих частин рівностей

$\operatorname{rhs}(\%)=-\operatorname{rhs}(\%)$ :

\# Елементарні перетворення

$\operatorname{lhs}(\%) * \operatorname{rho}^{\wedge}(2 * m+1) / C[1]=\operatorname{rhs}(\%) * \operatorname{rho}^{\wedge}(2 * m) * \operatorname{rho} / C[1]:$

$\operatorname{lhs}(\%)-\operatorname{rhs}(\%)=0$ :

$\operatorname{collect}(\%,[\operatorname{diff}(\mathrm{G}[1]($ alpha),alpha)]);

$$
\begin{gathered}
\frac{\partial^{2}}{\partial \rho \partial \alpha} \sigma_{0}(\rho, \alpha)=-\frac{\left((-2 m+2)\left(\frac{d}{d \alpha} G_{1}(\alpha)\right)+\left(\frac{d^{2}}{d \alpha^{2}} G_{2}(\alpha)\right)\right) C_{1}}{\rho^{(2 m+1)}}, \\
\frac{\partial^{2}}{\partial \rho \partial \alpha} \sigma_{0}(\rho, \alpha)=\frac{2\left((-2 m+2) G_{2}(\alpha)-\left(\frac{d}{d \alpha} G_{1}(\alpha)\right)\right) C_{1} m}{\rho^{(2 m)} \rho}, \\
(-4 m+2)\left(\frac{d}{d \alpha} G_{1}(\alpha)\right)+\left(\frac{d^{2}}{d \alpha^{2}} G_{2}(\alpha)\right)+2(-2 m+2) G_{2}(\alpha) m=0 .
\end{gathered}
$$

Останнє нелінійне звичайне диференціальне рівняння другого порядку відносно двох невідомих функцій $G_{1}(\alpha), G_{2}(\alpha)$ з урахуванням уведених позначень тотожне отриманому в [1] із застосуванням іншого прийому, що базується на використанні співвідношення (37).

Співвідношення (25) перепишемо з урахуванням (12), (29)

$$
\left.\begin{array}{l}
\dot{\xi}_{\rho}=\frac{1}{2} \cdot \frac{g(\alpha)}{\rho^{2}} \cdot \cos (2 \cdot \varphi(\alpha)) \\
\dot{\xi}_{t}=-\frac{1}{2} \cdot \frac{g(\alpha)}{\rho^{2}} \cdot \cos (2 \cdot \varphi(\alpha)) \\
\dot{\gamma}=\frac{g(\alpha)}{\rho^{2}} \cdot \sin (2 \cdot \varphi(\alpha))
\end{array}\right\} .
$$

На основі співвідношення (2) запишемо умову сумісності деформацій

$$
\frac{\partial \dot{\xi}_{t}}{\partial \alpha}=\dot{\gamma} .
$$
вигляду

Друге диференціальне рівняння отримаємо із використанням цієї умови, яка з урахуванням (41) набуде

$$
\frac{\partial}{\partial \alpha}(g(\alpha) \cdot \cos (2 \cdot \varphi(\alpha)))+2 \cdot g(\alpha) \cdot \sin (2 \cdot \varphi(\alpha))=0 .
$$

У [1] підкреслюється, що уведені припущення надали можливість звести розв'язання поставленої задачі до чисельного інтегрування системи диференціальних рівнянь (40), (43).

Для цього необхідно визначити крайові умови. Очевидно, що на осі симетрії, що задається рівністю $\alpha=0$, дотичне напруження $\tau=0$, отже, відповідно до співвідношень $(31) G_{2}(0)=0$, тобто $g^{m}(0) \cdot \sin (2 \cdot \varphi(0))=0$. Це означає, що принаймні одне із значень $g(0), \varphi(0)$ має дорівнювати нулю. Оскільки інтенсивність швидкостей деформацій $\dot{\xi}_{i}$ та інтенсивність напружень $\sigma_{i}$ відмінні від нуля на осі симетрії, то, відповідно до (12), (22), $g(0) \neq 0$. Тоді має виконуватися рівність $\sin (\varphi(0))=0$, звідки випливає $\varphi(0)=0$.

Для отримання другої крайової умови приймається, що на стінках матриці дотичне напруження $\tau$ досягає свого максимуму. Отже, при $\alpha=\alpha_{1}$, відповідно до співвідношень (31) $G_{2}\left(\alpha_{1}\right)=\left(G_{2}\right)_{\max }$, звідки, за аналогією 3 міркуваннями стосовно першої крайової умови, $\sin \left(2 \cdot \varphi\left(\alpha_{1}\right)\right)=1$, або $\varphi\left(\alpha_{1}\right)=\pi / 4$.

Той самий результат для $\varphi\left(\alpha_{1}\right)$ випливає із співвідношень $(6),(26)$ за припущенням прилипання на стінках матриці [1]. 
У праці [1] зазначається, що виключенням функції $g(\alpha)$ із диференціальних рівнянь (40), (43) отримуємо нелінійне диференціальне рівняння другого порядку відносно функції $\varphi=\varphi(\alpha)$

$$
\frac{d^{2} \varphi}{d \alpha^{2}}+F_{1} \cdot\left(\frac{d \varphi}{d \alpha}\right)^{2}+F_{2} \cdot \frac{d \varphi}{d \alpha}+F_{3}=0 .
$$

де

$$
\begin{gathered}
\Psi=\frac{2 \cdot \operatorname{tg}(2 \cdot \varphi(\alpha))}{m \cdot \operatorname{tg}^{2}(2 \cdot \varphi(\alpha))+1}, \\
F_{1}=\Psi \cdot\left([m \cdot \operatorname{tg}(2 \cdot \varphi(\alpha))]^{2}+\frac{m}{\cos ^{2}(2 \cdot \varphi(\alpha))}+2 \cdot m-1\right), \\
F_{2}=-\Psi \cdot\left(2 \cdot[m \cdot \operatorname{tg}(2 \cdot \varphi(\alpha))]^{2}+\frac{m}{\cos ^{2}(2 \cdot \varphi(\alpha))}+2 \cdot m^{2}-m+1\right), \\
F_{3}=m^{2} \cdot \Psi \cdot\left[1+\operatorname{tg}^{2}(2 \cdot \varphi(\alpha))\right] .
\end{gathered}
$$

Отримання на основі (40), (43) диференціального рівняння (44) не є тривіальним і супроводжується громіздкими символьними перетвореннями, тому розглянемо виконання цих операцій із використанням СКМ Maple.

Розпишемо похідну у (43) та знайдемо вираз, що використовуватимемо далі

$\operatorname{diff}(g($ alpha $) * \cos (2 *$ phi(alpha $))$, alpha $)+2 * g($ alpha $) * \sin (2 *$ phi(alpha $))=0$;

\# Ділимо попереднс рівняння на g(alpha)* $\sin (2 *$ phi(alpha))

$\operatorname{map}(z->z / g($ alpha $) / \cos (2 *$ phi(alpha) $), \operatorname{lhs}(\%))=0$;

\# Розв'язуємо попереднє рівняння відносно потрібного нам виразу

isolate(\%,diff(g(alpha),alpha)/g(alpha));

\# Здійснюсмо елементарні перетворення

$\operatorname{lhs}(\%)=$ convert $(\operatorname{rhs}(\%), \tan )$;

$\operatorname{lhs}(\%)=\operatorname{map}(\mathrm{z}->\mathrm{z} / 2, \operatorname{rhs}(\%))$ :

$\operatorname{lhs}(\%)=2 * \operatorname{collect}(\operatorname{rhs}(\%), \tan )$;

dg_g: $=\%$ :

$$
\begin{aligned}
\left(\frac{d}{d \alpha} \mathrm{g}(\alpha)\right) \cos (2 \phi(\alpha))-2 \mathrm{~g}(\alpha) \sin (2 \phi(\alpha))\left(\frac{d}{d \alpha} \phi(\alpha)\right) & \\
+2 \mathrm{~g}(\alpha) \sin (2 \phi(\alpha)) & =0 \\
\frac{\frac{d}{d \alpha} \mathrm{g}(\alpha)}{\mathrm{g}(\alpha)} & =2\left(\left(\frac{d}{d \alpha} \phi(\alpha)\right)-1\right) \tan (2 \phi(\alpha))
\end{aligned}
$$

Знайдемо $\frac{d}{d \alpha}\left(G_{1}(\alpha)\right)$ та $\frac{d}{d \alpha}\left(G_{2}(\alpha)\right)$

$\operatorname{Diff}(G[1]($ alpha),alpha) $=\operatorname{diff}(G 1$, alpha);d1G1:=rhs(\%):

$\operatorname{lhs}(\% \%)=g(\text { alpha })^{\wedge} m^{*} \cos (2 *$ phi(alpha $\left.)\right)^{*} \operatorname{map}\left(z-\operatorname{convert}\left(z /\left(g(\right.\right.\right.$ alpha $) \wedge m^{*} \cos (2 *$ phi(alpha $\left.\left.\left.\left.)\right)\right), \tan \right), \mathrm{d} 1 G 1\right) ;$ eq50:=\%:

$$
\begin{gathered}
\frac{d}{d \alpha} G_{1}(\alpha)= \\
\frac{\mathrm{g}(\alpha)^{m} m\left(\frac{d}{d \alpha} \mathrm{g}(\alpha)\right) \cos (2 \phi(\alpha))}{\mathrm{g}(\alpha)}-2 \mathrm{~g}(\alpha)^{m} \sin (2 \phi(\alpha))\left(\frac{d}{d \alpha} \phi(\alpha)\right) \\
\mathrm{g}(\alpha)^{m} \cos (2 \phi(\alpha))\left(\frac{m\left(\frac{d}{d \alpha} \mathrm{g}(\alpha)\right)}{\mathrm{g}(\alpha)}-2\left(\frac{d}{d \alpha} \phi(\alpha)\right) \tan (2 \phi(\alpha))\right)
\end{gathered}
$$

Diff(G[2](alpha),alpha) $=\operatorname{diff}(G 2$, alpha);d1G2:=rhs(\%):

$\operatorname{lhs}(\% \%)=g(\text { alpha })^{\wedge} m * \cos (2 *$ phi(alpha $\left.)\right) * \operatorname{map}\left(z-\operatorname{convert}\left(z /\left(g(\operatorname{alpha}) \wedge m^{*} \cos (2 *\right.\right.\right.$ phi(alpha $\left.\left.\left.\left.)\right)\right), \tan \right), d 1 G 2\right)$; eq51:=\%: 


$$
\begin{gathered}
\frac{d}{d \alpha} G_{2}(\alpha)= \\
\frac{\mathrm{g}(\alpha)^{m} m\left(\frac{d}{d \alpha} \mathrm{g}(\alpha)\right) \sin (2 \phi(\alpha))}{\mathrm{g}(\alpha)}+2 \mathrm{~g}(\alpha)^{m} \cos (2 \phi(\alpha))\left(\frac{d}{d \alpha} \phi(\alpha)\right) \\
\frac{d}{d \alpha} G_{2}(\alpha)= \\
\mathrm{g}(\alpha)^{m} \cos (2 \phi(\alpha))\left(\frac{m\left(\frac{d}{d \alpha} \mathrm{g}(\alpha)\right) \tan (2 \phi(\alpha))}{\mathrm{g}(\alpha)}+2\left(\frac{d}{d \alpha} \phi(\alpha)\right)\right) .
\end{gathered}
$$

Перепишемо співвідношення (50), (51) з використанням заміни відповідно до (49). Ця заміна є ключовим прийомом виключення із системи нелінійних диференціальних рівнянь невідомої функції $g(\alpha)$ $\operatorname{subs}\left(m * d g \_g\right.$, eq50);

$\operatorname{lhs}(\%)=\operatorname{collect}(\operatorname{rhs}(\%),[\tan , \operatorname{diff}($ phi(alpha),alpha),g(alpha)]);

$\operatorname{lhs}(\%)=\operatorname{simplify}(2 * \mathrm{~g}($ alpha $) \wedge \mathrm{m} * \cos (2 *$ phi(alpha $)) * \operatorname{op}(2, \operatorname{rhs}(\%)), \operatorname{trig}) * \operatorname{map}(\mathrm{z}-$ $>\operatorname{simplify}\left(\mathrm{z} /\left(2 * \mathrm{~g}(\text { alpha })^{\wedge} \mathrm{m}^{*} \cos (2 *\right.\right.$ phi(alpha $\left.\left.\left.)\right)\right)\right)$, op(1,rhs(\%)));D1G1:=rhs(\%):

$$
\begin{aligned}
& \frac{d}{d \alpha} G_{1}(\alpha)=g(\alpha)^{m} \cos (2 \phi(\alpha)) \\
& \left(2 m\left(\left(\frac{d}{d \alpha} \phi(\alpha)\right)-1\right) \tan (2 \phi(\alpha))-2\left(\frac{d}{d \alpha} \phi(\alpha)\right) \tan (2 \phi(\alpha))\right) \\
& \frac{d}{d \alpha} G_{1}(\alpha)= \\
& \left(g(\alpha)^{m} \cos (2 \phi(\alpha))(2 m-2)\left(\frac{d}{d \alpha} \phi(\alpha)\right)-2 \mathrm{~g}(\alpha)^{m} \cos (2 \phi(\alpha)) m\right) \\
& \tan (2 \phi(\alpha)) \\
& \frac{d}{d \alpha} G_{1}(\alpha)=2 \mathrm{~g}(\alpha)^{m} \sin (2 \phi(\alpha))\left((m-1)\left(\frac{d}{d \alpha} \phi(\alpha)\right)-m\right) .
\end{aligned}
$$

$\operatorname{subs}(m * \tan (2 *$ phi(alpha $)) * d g \_g$, eq51):

$\operatorname{lhs}(\%)=2 * o p(1, \operatorname{rhs}(\%)) * o p(2, \operatorname{rhs}(\%)) * \operatorname{collect}(o p(3, \operatorname{rhs}(\%)) / 2, \operatorname{diff}(\operatorname{phi}(\operatorname{alpha})$, alpha $))$;

G3:=select(has, $\operatorname{rhs}(\%)$, tan):

$\operatorname{lhs}(\% \%)=2 * G[1] * G[3]$;

$\mathbf{G}[\mathbf{3}]=\mathbf{G 3}$;

$$
\begin{gathered}
\frac{d}{d \alpha} G_{2}(\alpha)=2 \mathrm{~g}(\alpha)^{m} \cos (2 \phi(\alpha)) \\
\left(\left(m \tan (2 \phi(\alpha))^{2}+1\right)\left(\frac{d}{d \alpha} \phi(\alpha)\right)-m \tan (2 \phi(\alpha))^{2}\right) \\
\frac{d}{d \alpha} G_{2}(\alpha)=2 G_{1} G_{3}, \\
G_{3}=\left(m \tan (2 \phi(\alpha))^{2}+1\right)\left(\frac{d}{d \alpha} \phi(\alpha)\right)-m \tan (2 \phi(\alpha))^{2} .
\end{gathered}
$$

Знайдемо другу похідну

$$
\begin{aligned}
& \frac{d^{2}}{d \alpha^{2}}\left(G_{2}(\alpha)\right)=\frac{d}{d \alpha}\left(2 \cdot G_{1}(\alpha) \cdot G_{3}(\alpha)\right)= \\
& =2 \cdot\left(G_{3}(\alpha) \cdot \frac{d}{d \alpha}\left(G_{1}(\alpha)\right)+G_{1}(\alpha) \cdot \frac{d}{d \alpha}\left(G_{3}(\alpha)\right)\right)
\end{aligned}
$$

Обчислення та перетворення виразу для похідної $\frac{d}{d \alpha}\left(G_{3}(\alpha)\right)$ здійснимо за допомогою СКМ Maple $\operatorname{Diff}(G[3]($ alpha),alpha) $=\operatorname{diff}(G 3$, alpha);

$\operatorname{lhs}(\%)=\operatorname{collect}(\operatorname{rhs}(\%),[\operatorname{diff}($ phi(alpha), $\$($ alpha,2)),diff(phi(alpha),alpha)]); d1G3:=rhs(\%): 


$$
\begin{gathered}
\frac{d}{d \alpha} G_{3}(\alpha)=4 m \tan (2 \phi(\alpha))\left(1+\tan (2 \phi(\alpha))^{2}\right)\left(\frac{d}{d \alpha} \phi(\alpha)\right)^{2} \\
+\left(m \tan (2 \phi(\alpha))^{2}+1\right)\left(\frac{d^{2}}{d \alpha^{2}} \phi(\alpha)\right) \\
-4 m \tan (2 \phi(\alpha))\left(1+\tan (2 \phi(\alpha))^{2}\right)\left(\frac{d}{d \alpha} \phi(\alpha)\right) \\
\frac{d}{d \alpha} G_{3}(\alpha)=4 m \tan (2 \phi(\alpha))\left(1+\tan (2 \phi(\alpha))^{2}\right)\left(\frac{d}{d \alpha} \phi(\alpha)\right)^{2} \\
+\left(m \tan (2 \phi(\alpha))^{2}+1\right)\left(\frac{d^{2}}{d \alpha^{2}} \phi(\alpha)\right) \\
-4 m \tan (2 \phi(\alpha))\left(1+\tan (2 \phi(\alpha))^{2}\right)\left(\frac{d}{d \alpha} \phi(\alpha)\right)
\end{gathered}
$$

Рівняння (40), записане в середовищі СКМ Maple з урахуванням уведених позначень матиме вигляд $2 *(1-2 * m) * D 1 G 1+2 *(G 3 * D 1 G 1+G 1 * d 1 G 3)+4 * m *(1-m) * G 2=0$ :

\# Зведемо подібні

$\operatorname{collect}(\%,[\operatorname{diff}($ phi(alpha),alpha\$2),diff(phi(alpha),alpha)]);

$$
\begin{aligned}
& 2\left(m \tan (2 \phi(\alpha))^{2}+1\right) \mathrm{g}(\alpha)^{m} \cos (2 \phi(\alpha))\left(\frac{d^{2}}{d \alpha^{2}} \phi(\alpha)\right)+( \\
& 4\left(m \tan (2 \phi(\alpha))^{2}+1\right) \mathrm{g}(\alpha)^{m} \sin (2 \phi(\alpha))(m-1) \\
& \left.+8 m \tan (2 \phi(\alpha))\left(1+\tan (2 \phi(\alpha))^{2}\right) \mathrm{g}(\alpha)^{m} \cos (2 \phi(\alpha))\right) \\
& \left(\frac{d}{d \alpha} \phi(\alpha)\right)^{2}+\left(-4 m \tan (2 \phi(\alpha))^{2} \mathrm{~g}(\alpha)^{m} \sin (2 \phi(\alpha))(m-1)\right. \\
& -4\left(m \tan (2 \phi(\alpha))^{2}+1\right) \mathrm{g}(\alpha)^{m} \sin (2 \phi(\alpha)) m \\
& -8 m \tan (2 \phi(\alpha))\left(1+\tan (2 \phi(\alpha))^{2}\right) \mathrm{g}(\alpha)^{m} \cos (2 \phi(\alpha)) \\
& \left.+4(1-2 m) \mathrm{g}(\alpha)^{m} \sin (2 \phi(\alpha))(m-1)\right)\left(\frac{d}{d \alpha} \phi(\alpha)\right) \\
& -4(1-2 m) \mathrm{g}(\alpha)^{m} \sin (2 \phi(\alpha)) m+4 m(1-m) \mathrm{g}(\alpha)^{m} \sin (2 \phi(\alpha)) \\
& +4 m^{2} \tan (2 \phi(\alpha))^{2} \mathrm{~g}(\alpha)^{m} \sin (2 \phi(\alpha))=0
\end{aligned}
$$

Після ділення обох частин рівняння на $g(\alpha)^{m} \cdot \cos (2 \cdot \varphi(\alpha))$ та інших нескладних перетворень, які зручніше проводити в напівавтоматизованому режимі використання СКМ, отримаємо

де

$$
\frac{d^{2} \varphi}{d \alpha^{2}}+\Psi \cdot f_{1} \cdot\left(\frac{d \varphi}{d \alpha}\right)^{2}+\Psi \cdot f_{2} \cdot \frac{d \varphi}{d \alpha}+F_{3}=0 .
$$

$$
\begin{gathered}
f_{1}=m \cdot(1+m) \cdot \operatorname{tg}^{2}(2 \cdot \varphi(\alpha))+3 \cdot m-1, \\
f_{2}=-\left(m \cdot(2 \cdot m+1) \cdot \operatorname{tg}^{2}(2 \cdot \varphi(\alpha))+2 \cdot m^{2}+1\right),
\end{gathered}
$$

За допомого СКМ Maple неважко перевірити, що рівняння (44), з урахуванням позначень (45), (46), (47), (48) тотожно рівнянню (57) з урахуванням позначень (58), (59)

$m *(1+m) * \tan (2 * p h i) \wedge 2+3 * m-1$ :

$m^{\wedge} 2 * \tan (2 *$ phi $) \wedge 2+m / \cos (2 * \text { phi })^{\wedge} 2+2 * m-1$ :

simplify(\%\%-\%);

$-\left(m *(2 * m+1) * \tan (2 * \text { phi })^{\wedge} 2+2 * m^{\wedge} 2+1\right):$

$-\left(2 * m^{\wedge} 2 * \tan (2 * \text { phi })^{\wedge} 2+m / \cos (2 * \text { phi })^{\wedge} 2+2 * m^{\wedge} 2-m+1\right):$

simplify $(\% \%-\%)$;

\section{Висновки}

Застосування сучасного математичного інструментарію - CКМ Maple, а також сучасних показників напружено-деформованого стану матеріалів надало можливість удосконалити метод розв'язання двовимірної задачі пресування штаби на основі аналізу побудови математичної моделі у вигляді системи двох звичайних 
нелінійних диференціальних рівнянь. Розроблені інформаційні Марlе-технології є складовими, як методики аналізу побудови математичної моделі пресування штаби, так і комп'ютерної підтримки моделювання вказаного процесу для широкого спектра технологічних параметрів та властивостей матеріалів. Запропонований підхід надає можливості більш ефективного та широкого використання відомих методів визначення напружено-деформованого стану матеріалів, що уже стали класичними. 3 одного боку, в умовах відсутності доступу до обчислювальних комплексів типу DEFORM 3D, це надає можливість теоретичного дослідження технологічних процесів, а 3 іншого $є$ джерелом отримання тестових задач для апробації достовірності результатів, що отримуються за допомогою зазначених обчислювальних комплексів.

Аннотация. Проанализирован метод решения двумерной задачи прессования полосы с иелью усовериенствования и разработки компьютерной поддержки его использования. В результате исследований в рамках модели нелинейно-вязкого тела была установлена структура зависимостей интенсивности напряжений, накопленной деформации и безразмерного показателя напряженного состояния от радиуса и полярного угла. Предложены новые приемы получения известных зависимостей в виде системы двух обычных нелинейных дифференциальных уравнений и сведения этой системы к нелинейному дифференциальному уравнению 2-го порядка с переменными коэффициентами. Разработаны соответствуюшие информационные Maple-технологии.

Ключевые слова: прессования полосы, двумерная задача, напряженно-деформированное состояние, накопленная деформация, уравнение состояния.

\begin{abstract}
A method for solving a two-dimensional strip pressing problem was analyzed in order to improve the development and support of computer use of it. As a result of research in the framework of a model nonlinear viscoelastic body was got structure the dependencies stress intensity, accumulated deformation and dimensionless stress state index from the radius and the polar angle. New techniques for the preparation of known dependencies as a system of two ordinary nonlinear differential equations have been proposed. The reduction of this system to a nonlinear differential equation of 2 nd order with variable coefficients has been shown. Corresponding information Maple-technologies have been developed.
\end{abstract}

Keywords: strip compression, two-dimensional problem, the stress-strain state, the accumulated strain, the equation of state.

\title{
Бібліографічний список використаної літератури
}

1. Малинин Н. Н. Ползучесть в обработке материалов / Н. Н. Малинин. - М. : Машиностроение, 1986. - 216 с.

2. Соколовский В. В. Теория пластичности / В. В. Соколовский. - М. : «Высшая школа», 1969. - 608 с.

3. Огородников В. А. Оценка деформируемости металлов при обработке давлением / В. А. Огородников - К.: Выща шк., 1983. $-173 \mathrm{c}$.

4. Матвийчук $B . A$. Совершенствование процессов локальной ротационной обработки давлением на основе анализа деформируемости металлов: монография / В. А. Матвийчук, И. С. Алиев. - Краматорск: ДГМА, 2009. - 268 с. ISBN 978-966-379-317-7.

5. Михалевич В. М. Тензорні моделі накопичення пошкоджень / В. М. Михалевич / Вінниця: "УНІВЕРСУМ- Вінниця", 1998 - $195 \mathrm{c}$.

6. Lebedev A. A. On the Choice of Stress Invariants in Solving Problems of Mechanics/ A. A. Lebedev, V. M. Mikhalevich // Strength of Materials N 35 (3), Plenum Publishing Corporation (USA), May - June, 2003, 217-224.

7. Mikhalevich V. M. Modeling of plastic deformation in a cylindrical specimen under edge compression / V. M. Mikhalevich, A. A. Lebedev, Yu Dobranyuk // Strength of Materials. - 2011, Vol. 43, No. 6, p. 591-603.

\section{References}

1. Malinin, N.N. (1986), Polzuchest' v obrabotke materialov [Creep in materials processing], Mashinostroenie, Moscow, Russian.

2. Sokolovskij, V.V. (1969), Teorija plastichnosti [The theory of plasticity], «Vysshaja shkola», Moscow, Russian.

3. Ogorodnikov, V.A. (1983), Ocenka deformiruemosti metallov pri obrabotke davleniem [Evaluation of metal deformability during processing by the pressure], Vyshha shk, Kyiv, Ukraine.

4. Matvijchuk, V.A . and Aliev, I.S. (2009), Sovershenstvovanie processov lokal'noj rotacionnoj obrabotki davleniem na osnove analiza deformiruemosti metallov: monografija [Improvement of local rotary pressure processing on the basis of metal deformability analysis], , DGMA, Kramatorsk, ISBN 978-966-379-317-7.

5. Mihalevich, V.M. (1998), Tenzorni modeli nakopichennja poshkodzhen' [Tensor model of damage accumulation], "UNIVERSUM, Vinnicja", Vinnicja, Ukraine.

6. Lebedev, A.A. and Mikhalevich, V.M. (2003), On the Choice of Stress Invariants in Solving Problems of Mechanics, Strength of Materials, no 35 (3), Plenum Publishing Corporation (USA), May - June, 2003, 217-224.

7. Mikhalevich, V.M., Lebedev, A.A. and Dobranyuk, Yu (2011), Modeling of plastic deformation in a cylindrical specimen under edge compression, Strength of Materials, vol. 43, no. 6, pp. 591-603. 